\title{
Research Note \\ Deprojection of luminosity functions of galaxies in the Coma cluster
}

\author{
M. Beijersbergen, W. E. Schaap, and J. M. van der Hulst
}

Kapteyn Astronomical Institute, PO Box 800, 9700 AV Groningen, The Netherlands

Received 7 February 2002 / Accepted 15 May 2002

\begin{abstract}
We use a simple analytic model to deproject 2-d luminosity functions (LF) of galaxies in the Coma cluster measured by Beijersbergen et al. 2002. We demonstrate that the shapes of the LFs change after deprojection. It is therefore essential to correct LFs for projection effects. The deprojected LFs of the central area have best-fitting Schechter parameters of $M_{U}^{*}=$ $-18.31_{-0.08}^{+0.08}$ and $\alpha_{U}=-1.27_{-0.018}^{+0.018}, M_{B}^{*}=-19.79_{-0.15}^{+0.14}$ and $\alpha_{B}=-1.44_{-0.016}^{+0.016}$ and $M_{r}^{*}=-21.77_{-0.28}^{+0.20}$ and $\alpha_{r}=-1.27_{-0.012}^{+0.012}$. The corrections are not significant enough to change the previously observed trend of increasing faint end slopes with increasing distance to the cluster center. The weighted $U, B$, and $r$ band slopes of the deprojected LFs show a slightly weaker steepening with increasing projected cluster radius.
\end{abstract}

Key words. galaxies: clusters: individual: Coma (A1656) - galaxies: luminosity function - galaxies: evolution galaxies: formation

\section{Introduction}

In the past large field studies of the photometric properties of galaxies in the Coma cluster have been based on photographic plates (e.g. Godwin et al. 1983; Lugger 1989). More recent studies have been made with CCD detectors which provide higher photometric accuracy. However, a limitation of the use of CCDs has been the relatively small field of view. Consequently, these studies have been limited to very small areas, ranging from $\sim 50 \operatorname{arcmin}^{2}$ (e.g. Bernstein et al. 1995; Mobasher \& Trentham 1998) to $1500 \operatorname{arcmin}^{2}$ (e.g. Lobo et al. 1997), mainly focused on the cluster center. These small fields can not be used to study the dependence of the luminosity function (LF) on the projected distance from the cluster center. Furthermore, lacking the photometric data at larger distances from the core it is not possible to correct a central LF for projection effects. Accordingly, the published LFs of galaxies in the Coma cluster are all projected and are, therefore, luminosity distributions rather than actual LFs. It is important to test how well these correspond to the actual deprojected LFs.

Recently, Valotto et al. (2001) used numerical simulations to analyse systematic effects in the determination of the galaxy luminosity function in clusters. They find that projection effects conspire to produce artificially steep faint end slopes. Beijersbergen et al. (2002, hereafter BHDH) presented statistical $U, B$ and $r$ band projected LFs for galaxies in the Coma cluster in five annuli out to a projected radius of 1.4 degrees for the $B$ and $r$ bands and 0.74 degrees for the $U$ band. In total an

Send offprint requests to: M. Beijersbergen,

e-mail: beijersb@astro.rug.nl area of $5.2 \mathrm{deg}^{2}$ in $B$ and $r$ and $1.3 \mathrm{deg}^{2}$ in $U$ has been surveyed. This data offers the interesting possibility to deproject actually observed LFs and to obtain true 3-d LFs.

In this Research Note we use the information of all annuli to deproject the 2-d LFs. Our study extends the work in BHDH by inferring for the first time deprojected LFs as a function of projected distance from the cluster center. In addition, we study how the shapes of the LFs are affected by projection by measuring the faint end slopes as a function of distance to the cluster center and compare this with results from numerical simulations. Throughout this Research Note the projected LFs are taken from BHDH and are called 2-d LFs. Deprojected LFs are named 3-d LFs.

\section{Deprojection of luminosity functions}

Extensive literature exists on the uncovering of threedimensional structure from observations of projected quantities (see e.g. Zaroubi et al. 1998 and references therein). Usually methods have been described which were designed for a specific type of observation, in particular X-ray measurements. Here we use a very simple, analytic geometric deprojection scheme (Fabian et al. 1981). This scheme has mainly been used for the analysis of X-ray observations, but in principle it is suitable for any type of observations. We use this projection scheme to deproject the observed 2-d luminosity distributions of the galaxies in the Coma cluster to real, 3-d, luminosity functions. This scheme consists of assuming that the galaxies are symmetrically distributed in a sphere. This sphere is divided up in to a number of concentric shells, in which the galaxy 


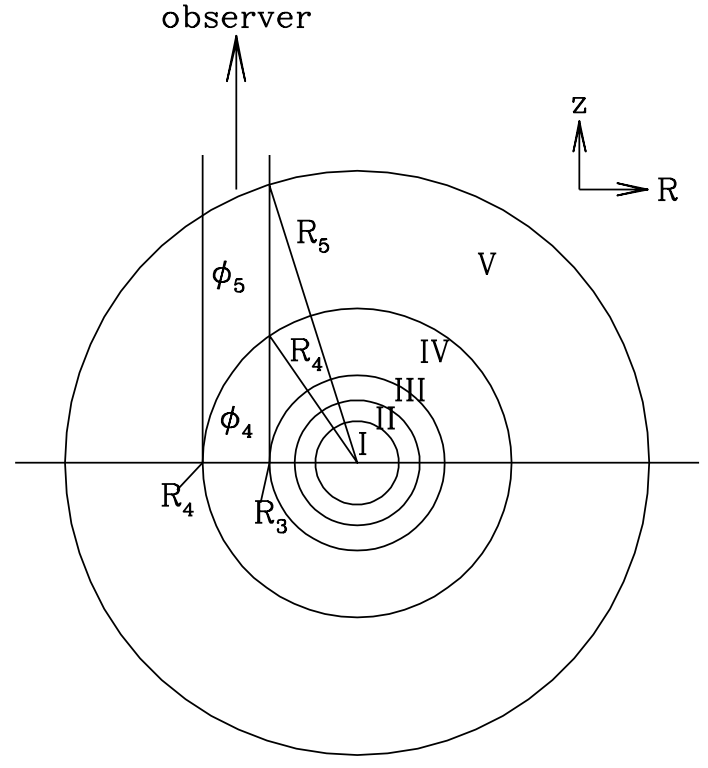

Fig. 1. Outline of the model used to deproject 2-d luminosity functions. The figure can be seen as a cut through a sphere and as its projection as seen by the observer. The five circles make up the projected annuli as used in Beijersbergen et al. (2002). Radii are I: $0-0.2$, II: $0.2-0.3$, III: $0.3-0.42$, IV: $0.42-0.74$ and V: $0.74-1.4$ degrees $\left(1^{\circ}=1.22 h_{100}^{-1} \mathrm{Mpc}\right.$ at Coma distance $)$.

density per magnitude is assumed to be constant. The value of the 3-d luminosity function in a particular shell can then be found by subtracting the contributions from the other shells due to projection. In Fig. 1 we show an outline of this model.

The number of projected galaxies per magnitude $m, N_{i, m}$, in annulus $i$, and the galaxy density per magnitude, $\phi_{m}$, are related as:

$$
\begin{aligned}
N_{i, m}= & \phi_{i, m} F\left(R_{i}, R_{i-1}, R_{i}\right) \\
& +\sum_{j=i+1}^{5} \phi_{j, m}\left\{\left[F\left(R_{j}, R_{i-1}, R_{i}\right)-F\left(R_{j-1}, R_{i-1}, R_{i}\right)\right]\right\}
\end{aligned}
$$

where $R_{i}$ are the radii of the annuli and the function $F$ is defined by:

$$
F(\alpha, \beta, \gamma) \equiv \frac{4}{3} \pi \alpha^{3}\left[\left(1-\beta^{2} / \alpha^{2}\right)^{3 / 2}-\left(1-\gamma^{2} / \alpha^{2}\right)^{3 / 2}\right]
$$

With the available 2-d information we can solve for all $\phi_{i, m}$, i.e. the true 3-d LFs. The $N_{i, m}$ are dependent on the areas of the annuli which are accurately known. However, the outermost annuli are not entirely filled by data: annulus $\mathrm{V}$ is filled for $\sim 80 \%$ in $B$ and $r$ and annulus IV is filled for $\sim 45 \%$ in $U$. We omitted annulus IV for the $U$ band and corrected $N_{5, m}$ for the $B$ and $r$ bands by assuming a constant projected density throughout this annulus. The function $F$ is solely dependent on geometry and has values of order $10^{-3}$ to $10^{-1}$. Figures 2 and 3 show both the 2-d (open symbols) and 3-d (solid symbols) LFs for all bands and annuli (all magnitudes are corrected for atmospheric extinction). The error bars of the 2-d LFs are not shown for clarity, but are somewhat smaller than the 3-d error bars. The 2-d errors are estimates of the uncertainty in the subtraction of the number of field galaxies. These estimates are based on very large control fields and therefore very accurate (see BHDH for an extensive discussion). The 3-d errors have been calculated using Eq. (1). The corresponding errors are therefore dependent on each other and fully determined by the uncertainty in the subtraction of the number of field galaxies. The vertical scales represent volume densities and the 2-d LFs are scaled as to match the bright ends of the 3-d LFs. This simplifies the comparison of their shapes. In annulus $\mathrm{V}$ the galaxy density is much lower than in the center, but still higher than in the field. That is to say, the derived 3-d $B$ and $r$ band LFs still suffer from (minor) projection effects due to cluster galaxies at radii even larger than $1.7 h_{100}^{-1} \mathrm{Mpc}$. The contamination by projection for the 3-d $U$ band LFs is larger since there we miss photometric information for radii larger than $0.5 h_{100}^{-1} \mathrm{Mpc}$.

\section{Discussion}

In the literature LFs of galaxies in clusters are usually given in units of number of galaxies per magnitude per area. What actually is measured is a luminosity distribution, denoted here as 2-d LF. A luminosity function of a sample of galaxies has units of galaxies per magnitude per volume. For observations of the entire projected volume of a cluster the two have the same shape since for all luminosities the same volume is sampled. BHDH studied changes in the LF as a function of distance to the cluster center. However, they did not correct for the fact that fore- and background galaxies, residing in the outer parts of the cluster, are projected onto the central cluster regions. The question is how much their results have been influenced by this effect. Below we study how the shapes of the luminosity distributions given in BHDH are affected by projection effects.

In the outermost annulus the projected area is converted to volume through a factor depending on the adopted geometry (radii), i.e. for this annulus the shapes of the 2-d and 3d LFs remain identical. From all LFs of the inner annuli the contributions to the galaxy counts from surrounding shells is subtracted using the model described in Sect. 2. The impact on the resulting LFs can be seen in Figs. 2 and 3. When the 2-d and 3-d LFs are compared binwise the differences, generally, are small. Yet, the error bars become larger and it should be kept in mind that the scale is logarithmic. Our result can be explained by the small values of the $F$ integrals in Eq. (1) combined with the density profile of the cluster. In all expressions we find combinations of relatively high values of the integrals times low densities, and vice versa, resulting in a minor net correction term. However, this correction term is different for each magnitude bin causing the shapes of corrected and uncorrected LFs to be different. Below, we quantify the differences by studying the change in the shapes of the 3-d LFs as a function of position in the cluster. The results are compared to the exact same analysis for the 2-d LFs.

\subsection{Dependence of luminosity functions on radial distance from the cluster center}

BHDH found that the 2-d central LFs could be well represented by Schechter functions. In Fig. 2 the best-fitting Schechter (1976) functions of the 3-d LFs are shown as solid lines. 


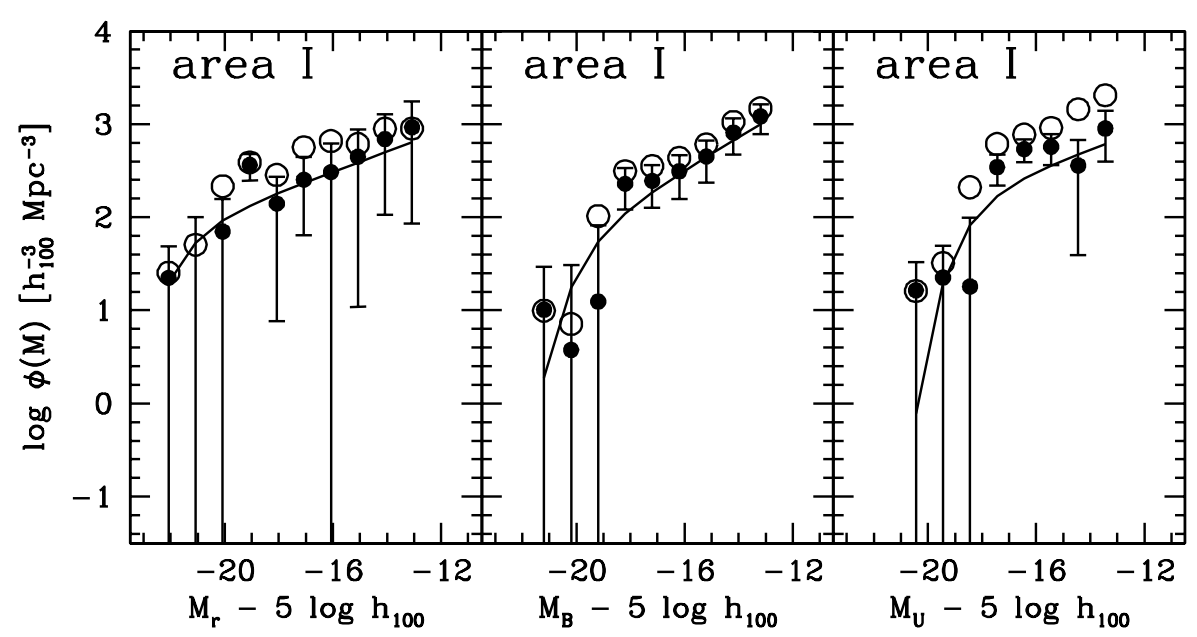

Fig. 2. Two and three dimensional luminosity functions of area I compared. Open symbols represent 2-d luminosity functions and solid symbols represent 3-d luminosity functions. The solid lines represent best-fitting Schechter functions to the 3-d data. For clarity the error bars of the 2-d luminosity functions are not shown.

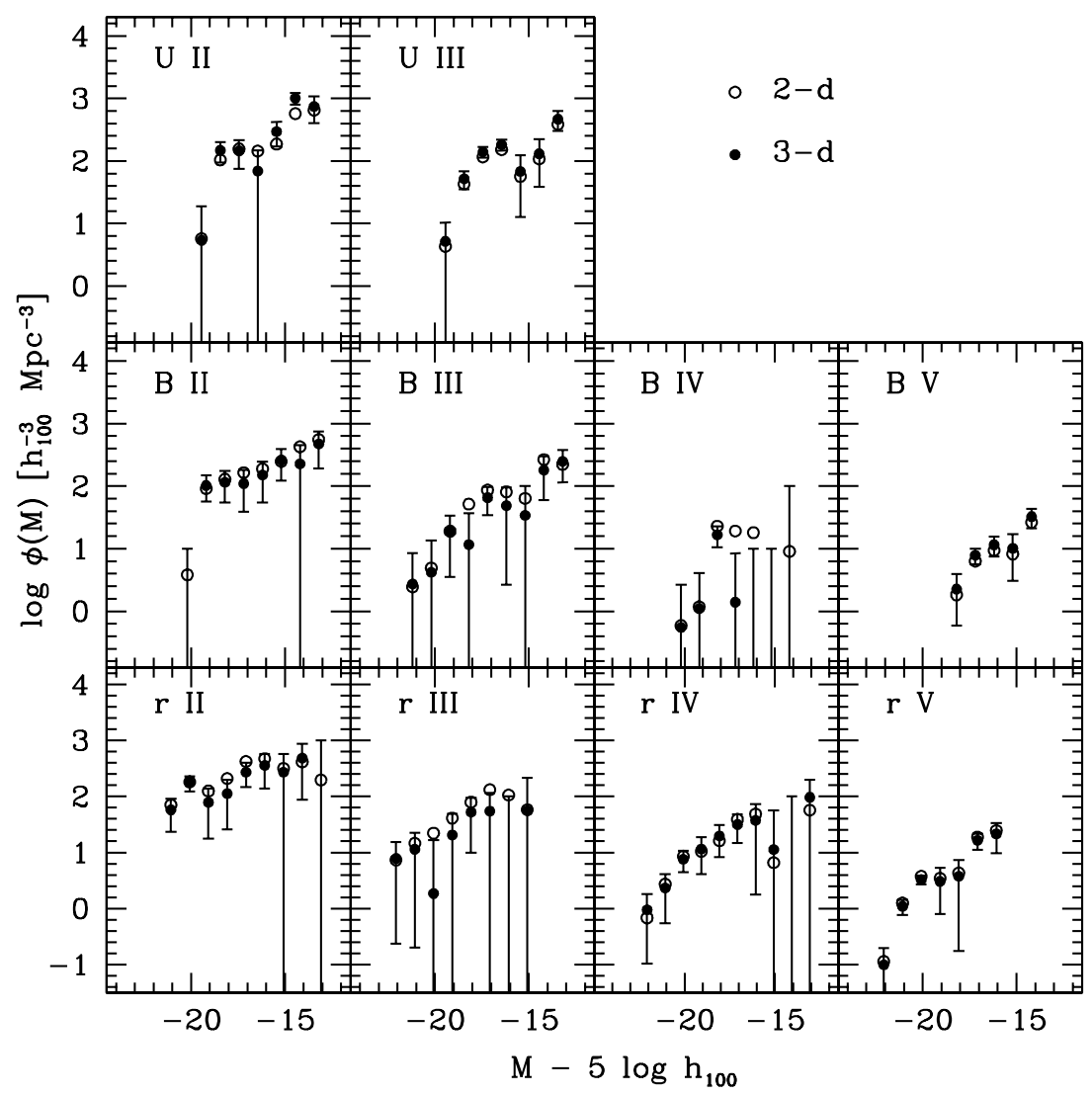

Fig. 3. Two and three dimensional luminosity functions of areas II to V compared. Open symbols represent 2-d luminosity functions and solid symbols represent 3-d luminosity functions. For clarity the error bars of the 2-d luminosity functions are not shown.

In Table 1 we compare the best-fitting Schechter parameters, $\alpha$ and $M^{*}$, for the 2-d and 3-d case. For the $r$ and $B$ bands the Schechter fits are again reasonable representations of the data, though the best-fitting parameters have changed. The $U$ band fit remains a poor representation of the data.

One of the main results of BHDH was the finding of a significant steepening of the faint end slopes with increasing cluster radius. A straight line fit $(s r+b)$, where $r$ is the cluster- centric radius, to the weighted $U, B$ and $r$ band slopes at each radius gives $s=0.11 \pm 0.02 \mathrm{Mpc}^{-1}$ for the 2-d LFs. In order to compare this with the situation where all 2-d LFs have been corrected for projection we fitted power laws $\left(b 10^{a M}\right)$ to the faint ends of the 3-d LFs in Figs. 2 and 3. The infalling group around NGC 4839 (Neumann et al. 2001) does not have a significant influence on any of the derived LFs. The LFs were fitted for $M_{r}>-20, M_{B}>-20$ and $M_{U}>-19$. We omitted 


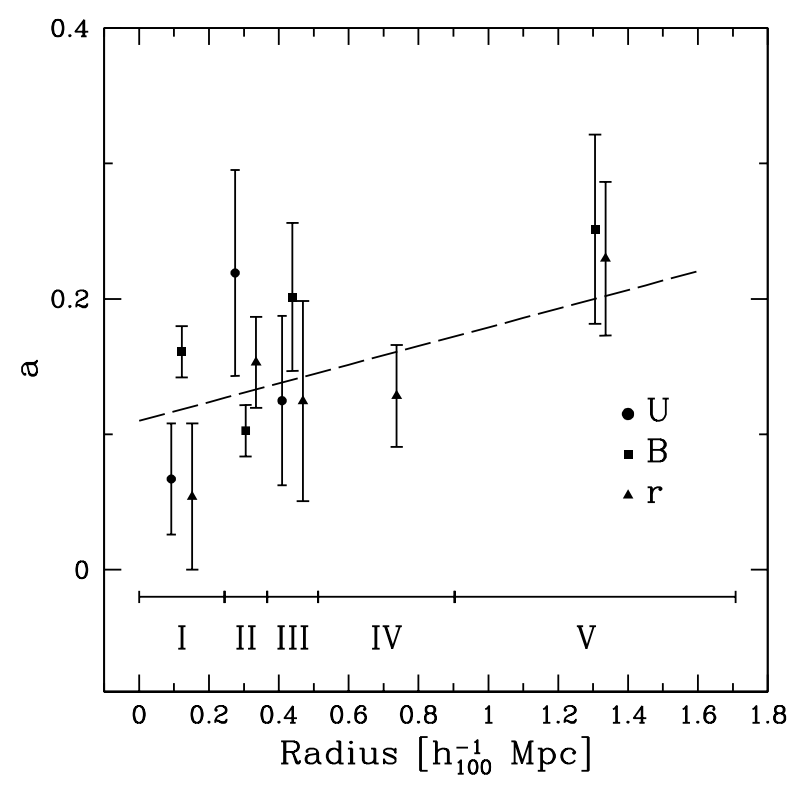

Fig. 4. Power law slopes $a$ as a function of cluster radius. The $U$ and $r$ band slopes are offset in radius by -0.03 and +0.03 , respectively. The faint ends of the LFs become steeper towards the cluster outskirts. The overall trend is indicated by the dashed line.

Table 1. Comparison of best-fitting Schechter parameters for 2-d and 3-d LFs. $M^{*}$ is not corrected for extinction.

\begin{tabular}{ccccc}
\hline \hline Filter & \multicolumn{2}{c}{2 2-d } & \multicolumn{2}{c}{$3-\mathrm{d}$} \\
& $\alpha$ & $M^{*}$ & $\alpha$ & $M^{*}$ \\
\hline$U$ & $-1.32_{-0.028}^{+0.018}$ & $-18.60_{-0.18}^{+0.13}$ & $-1.27_{-0.018}^{+0.018}$ & $-18.31_{-0.08}^{+0.08}$ \\
& & & & \\
$B$ & $-1.37_{-0.016}^{+0.024}$ & $-19.79_{-0.17}^{+0.18}$ & $-1.44_{-0.016}^{+0.016}$ & $-19.79_{-0.15}^{+0.14}$ \\
& & & & \\
$r$ & $-1.16_{-0.019}^{+0.012}$ & $-20.87_{-0.17}^{+0.12}$ & $-1.27_{-0.012}^{+0.012}$ & $-21.77_{-0.28}^{+0.20}$ \\
\hline
\end{tabular}

the slope of the $B$ band LF for area IV since there the LF is not well constrained. The results are shown in Fig. 4. A straight line fit to the weighted slopes of the 3 -d LFs at each radius gives $s=0.07 \pm 0.04 \mathrm{Mpc}^{-1}$ and is indicated by the dashed line. Although this is a somewhat weaker trend than derived from the 2-d results, it is different at $1 \sigma$ only and the trend is still clearly there.

Valotto et al. (2001) used a deep mock catalogue to analyse systematic effects in the determination of the galaxy luminosity function in clusters. They find that projection effects produce artificially steep faint end slopes. The cluster core suffers most from objects that are projected onto it. The LFs of area I show similar ( $r$ and $B$ band) or shallower ( $U$ band) faint end slopes when they are corrected for projection. We conclude that the impact of projection effects on the shapes of the 2-d LFs in Coma is not as severe as their simulations suggest. However, the 2-d LFs derived for relatively small areas suffer from projection effects and should therefore be properly corrected before one can reliably interpret their shapes.

\section{Summary and conclusions}

In this Research Note we have used a simple analytic model to deproject 2-d LFs of cluster galaxies. We have applied this to the LFs of the galaxies in the Coma cluster measured by BHDH. Under the assumptions of a spherically symmetric galaxy distribution and a constant galaxy density in each shell the 2-d LFs have been corrected for projection effects. The main results are summarized by Figs. 2 and 3. Projection effects affect the shapes of the 2-d LFs and in general make the faint end slopes artificially steeper, although the differences are small. The alterations are not significant enough as to arrive at different conclusions compared to those inferred from 2-d LFs. Furthermore, the previously derived steepening of the faint end slopes with increasing cluster radius is only slightly weakened when projection effects are taken into account. Overall, we have demonstrated by using a simple model that before interpreting shapes of 2-d LFs one needs to correct for projection.

Acknowledgements. We thank the referee for constructive comments.

\section{References}

Beijersbergen, M., Hoekstra, H., van Dokkum, P. G., \& van der Hulst, J. M. 2002, MNRAS, 329, 385 (BHDH)

Bernstein, G. M., Nichol, R. C., Tyson, J. A., Ulmer, M. P., \& Wittman, D. 1995, AJ, 110, 1507

Fabian, A. C., Hu, E. M., Cowie, L. L., \& Grindlay, J. 1981, ApJ, 248, 47

Godwin, J. G., Metcalfe, N., \& Peach, J. V. 1983, MNRAS, 202, 113

Lobo, C., Biviano, A., Durret, F., et al. 1997, A\&A, 317, 385

Lugger, P. M. 1989, ApJ, 343, 572

Mobasher, B., \& Trentham, N. D. 1998, MNRAS, 293, 315

Neumann, D. M., Arnaud, M., Gastaud, R., et al. 2001, A\&A, 365, L74

Schechter, P. 1976, ApJ, 203, 297

Valotto, C. A., Moore, B., \& Lambas, D. G. 2001, ApJ, 546, 157

Zaroubi, S., Squires, G., Hoffman, Y., \& Silk, J. 1998, ApJ, 500, L87 